\title{
INVESTIGACIONES
}

\section{Análisis de los determinantes del rendimiento académico. El caso de Contador Auditor de la Pontificia Universidad Católica de Valparaíso*}

\author{
Analysis of the determinants of academic performance. \\ The case of Auditor Accountant of the Pontifical Catholic University of Valparaíso
}

\section{Hanns de la Fuente-Mella ${ }^{a}$, Mercedes Marzo Navarro ${ }^{b}$, Carmen Berne-Manero ${ }^{b}$, Marta Pedraja Iglesias ${ }^{b}$ Camila González-Huenuman ${ }^{a}$}

\author{
${ }^{a}$ Pontificia Universidad Católica de Valparaíso, Facultad de Ciencias Económicas y Administrativas, \\ Escuela de Comercio. hanns.delafuente@pucv.cl, camila.gonzalezh@gmail.com \\ ${ }^{b}$ Universidad de Zaragoza, Facultad de Economía y Empresa, \\ Departamento de Dirección de Marketing e Investigación de Mercados. \\ mmarzo@unizar.es, cberne@unizar.es,mpedraja@unizar.es
}

\begin{abstract}
Este artículo plantea un modelo econométrico que permite determinar el número de semestres que invierte un estudiante de la carrera Contador Auditor de la Pontificia Universidad Católica de Valparaíso (Chile) en finalizar su titulación universitaria. Además, se trata de identificar los factores, relacionados con el rendimiento académico o no, que determinan dicha duración. Los resultados obtenidos muestran como variables de ambos tipos inciden en la duración de los estudios universitarios. En relación con las variables asociadas al rendimiento académico, las notas promedio en las asignaturas del área disciplinar finanzas y matemáticas, son las más influyentes en la duración de la carrera de Contador Auditor. Además, la edad, el tipo de establecimiento donde cursaron los estudios previos y el sexo de los estudiantes se han identificados como predictores válidos del rendimiento académico de los egresados.
\end{abstract}

Palabras claves: Duración Carrera, Rendimiento Académico, Determinantes Del Rendimiento, Egresados Universitarios; Modelamiento Econométrico.

\begin{abstract}
This article proposes an econometric model that allows to determine the number of semesters a student in the Accountant Auditor career of the Pontificia Universidad Católica de Valparaíso (Chile) invests in completing his university degree. In addition, it is about identifying the factors, related to academic performance or not, that determine this duration. The results obtained show how variables of both types affect the duration of university studies. In relation to the variables associated with academic performance, the average grades in the subject areas of finance and mathematics are the most influential in the duration of the career of Auditor Accountant. In addition, the age, the type of establishment where the previous studies were conducted, and the sex of the students have been identified as valid predictors of the academic performance of the graduates.
\end{abstract}

Keywords: Duration of Career, Academic Performance, Undergraduate Student, Econometric Modeling.

\footnotetext{
* Este trabajo ha sido financiado por el Gobierno de Aragón (Referencia Grupo CREVALOR) y cofinanciado con Feder 2014-2020 "Construyendo Europa desde Aragón". Así como al proyecto concedido por la Universidad de Zaragoza (UZ2018-SOC-04).
} 
Estudios Pedagógicos XLVII Nº 1: 469-482, 2021

ANÁLISIS DE LOS DETERMINANTES DEL RENDIMIENTO ACADÉMICO. EL CASO DE CONTADOR AUDITOR DE LA PONTIFICIA UNIVERSIDAD CATÓLICA DE VALPARAÍSO

\section{INTRODUCCIÓN}

Durante las últimas décadas el sistema educativo superior de Chile ha experimentado importantes transformaciones (Espinoza y Urzúa, 2015). En relación a su tasa de matrícula, apenas llegaba al 10\% a comienzos de la década de los 70 del pasado siglo, en la década de los 90 superaba el $20 \%$ y en el año 2000 alcanzó el 40\%. Concretamente en el año 2002 el número total de estudiantes matriculados ascendía aproximadamente a 1.100.000 estudiantes (Herrera, Pérez y Echeita, 2016; Yáñez y Soria, 2017). No sólo se ha producido un importante incremento en el número de estudiantes, sino también en la diversidad del perfil de los mismos. Así, se ha incrementado la presencia de grupos de estudiantes con características diferentes a las de quienes accedían tradicionalmente a los estudios superiores (Sebastián, 2007). De este modo, ya no solo la elite accede a la universidad, sino que también lo hace un grupo, más numeroso y diverso en cuanto a sus conocimientos, habilidades y destrezas (Himmel 2002, 2003). Por ello, sería necesario que las instituciones de Educación Superior se reorienten con el objetivo de mejorar su forma de atender la diversidad estudiantil (Fernández, Martínez-Conde y Melipillán, 2018).

Otra importante transformación del sistema educativo acontecida en las dos últimas décadas radica en el rápido crecimiento en la variedad de instituciones y programas que se ofrecen, en tanto en lo relativo a la diversidad de centros educacionales, como con el incremento en el número de carreras técnicas y universitarias. Muestra de ello son las 60 instituciones de estudios superiores existentes en Chile, las cuales se dividen principalmente en dos tipos: las llamadas "universidades tradicionales", pertenecientes al Consejo de Rectores de las Universidades Chilenas integrado por 18 universidades públicas del país y 9 universidades de carácter privado; y por otro lado las 34 "universidades privadas" restantes.

Ante la importante transformación experimentada por el sistema educativo superior chileno resulta relevante avanzar un paso más y profundizar en el análisis del rendimiento académico de los estudiantes. Los datos muestran la existencia de un elevado porcentaje de estudiantes que no terminan sus carreras universitarias (deserción), junto con otra parte que no lo hace en el plazo marcado en el plan de estudios (lentificación) (Mateo-Canedo, Canet-Juric y Andrés, 2015). Un estudio realizado por Gónzalez (2007) para el periodo 1999-2003, muestra como la eficiencia media de una titulación universitaria ${ }^{1}$ en el caso de Chile se situaba en 46,3. Para el caso de las universidades públicas era de 50 y para el de las privadas en 37 , y diferenciando según el sexo de titulado en 49 para las mujeres y 43 para los hombres. Los indicadores relacionados con la deserción, permanencia y graduación de los estudiantes universitarios se han convertido en un factor de vital importancia para las universidades chilenas, ya que dichos indicadores son utilizados para la rendición de cuentas, y también en iniciativas legislativas que los incorporan como indicadores de eficiencia docente, y como resultado de los mismos se establece una estructura de crédito para financiar los estudios universitarios. Estos datos relacionados con las tasas de titulación no sólo afectan a la institución educacional, sino que también afectan al estudiante y a su familia, quienes son los que deben costear los semestres extra de duración de la carrera (Améstica, Llinas, y Escardíbul, 2017). Para aquellos estudiantes que acceden

Eficiencia de titulación promedio, definida como la proporción de estudiantes "T" que se titulaba en un año "t", en comparación con la matricula nueva en primer año "N", en el tiempo correspondiente a una duración "d" de las carreras establecidas en los planes de estudios oficiales. 
a la educación superior, el emplear más tiempo del previsto en el plan de estudios en finalizar la titulación, además de significar una limitante al mundo laboral, también alteran sus planes de continuidad de estudios futuros, ya que aumenta la probabilidad de desertar y no volver a retomar sus estudios (Clark y Ramsay, 1990; Palominos et al., 2018). Otro agente afectado por esta situación es la propia sociedad. Así, el gobierno chileno, cada año otorga becas de arancel y de mantención a estudiantes que no cuentan con los recursos suficientes para poder autofinanciar sus estudios, por lo cual, la deserción es un golpe duro a los intereses del gobierno en pro de fomentar profesionales para el día de mañana, que puedan contribuir al país (Combey, 1993). Por ejemplo, en 2016 se entregaron más de 92.000 becas a estudiantes de primer año, de las cuales, más de 88.000 correspondían a becas de arancel, y el restante corresponde al crédito fondo solidario. Por todo ello, estos datos dejan entrever una situación que se configura como un problema complejo, que necesita ser abordado.

Un primer paso sería identificar los factores que determinan el rendimiento de los titulados. Algunos autores proponen que estas diferencias en el rendimiento pueden tener su origen en diversos aspectos, que se podrían agrupar en sociales, culturales y tecnológicos (Sayans, Vázquez y Bernal, 2018).

Ante esta problemática, este trabajo pretende ser una primera aproximación a la identificación y modelización de los elementos que determinan la cantidad de semestres que tarda un estudiante en finalizar sus estudios universitarios, como medida de rendimiento del alumno. Para ello, tras una breve revisión de literatura que permita identificar las variables que pueden determinar el tiempo de finalización de los estudios, se analiza el caso los titulados en Contador Auditor de la Pontificia Universidad Católica de Valparaíso (PUCV) en Chile.

\section{REVISIÓN DE LA LITERATURA}

A continuación, se presenta una breve revisión de la literatura relacionada con la evaluación del rendimiento de los estudiantes universitarios.

El concepto del rendimiento académico es un término complejo que es interpretado de forma diferente según el significado que tiene para las distintas audiencias implicadas (Tomas-Miguel, Exposito-Langa y Sempere-Castello, 2014). Por ello, se considera un concepto multidimensional, relativo y contextual, del que es difícil aportar un criterio que lo defina de forma univoca para todos los agentes implicados (González-López, 2004).

Sin embargo, y para operacionalizar con el concepto es necesario establecer una definición. Así, el rendimiento académico, se puede definir como la suma de factores complejos que permiten dar un valor al logro académico de un estudiante, generalmente medido de forma cuantitativa con el fin de reflejar el éxito o fracaso en el ámbito educacional (Pérez-Luño, Jerónimo y Sánchez-Vázquez, 2000; Vélez Van y Roa, 2005). Por lo tanto, el valor de los resultados académicos alcanzados representa un buen indicador para estimar el rendimiento académico, y se asume que las notas representan los logros en los diferentes componentes del aprendizaje en la vida académica. La literatura pone de manifiesto que el rendimiento académico del estudiante no se debe únicamente a una sola capacidad, sino que es el fruto de la combinación de diversos factores que actúan en, y desde, la persona que aprende (Tomas-Miguel, Exposito-Langa y Sempere-Castello, 2014). 
Estudios Pedagógicos XLVII N 1: 469-482, 2021

ANÁLISIS DE LOS DETERMINANTES DEL RENDIMIENTO ACADÉMICO. EL CASO DE CONTADOR AUDITOR DE LA PONTIFICIA UNIVERSIDAD CATÓLICA DE VALPARAÍSO

La literatura confirma la existencia de múltiples elementos interrelacionados que indicen en el rendimiento. Estos componentes, tanto internos como externos al estudiante, incluyen aspectos personales, académicos y sociales (Pérez et al., 2000; Vélez Van y Roa, 2005; Quispe, 2010, Garbanzo, 2007; Córdoba et al., 2011).

Partiendo de la perspectiva de que el rendimiento académico es un fenómeno multifactorial (Edel, 2003), o multicausal (Garbanzo, 2007), se pueden encontrar diversas variables que intentan explicarlo, ya sean factores internos o externos asociados al estudiante. Estas variables pueden agruparse en dos bloques: aquellas que están relacionadas con las características no académicas de los estudiantes, tales como nivel económico del grupo familiar, entorno socioeconómico y sociocultural (capital cultural), modalidad de colegio, nivel educativo del padre y de la madre, etc., y aquellas relativas a sus características académicas, como sus notas en las pruebas de admisión, su desempeño escolar, etc.

La revisión de la literatura muestra numerosas investigaciones que intentan determinar los factores de éxito académico de los estudiantes en la universidad teniendo presente diversas características sociodemográficas de los mismos. Algunos autores afirman que el género del estudiante es determinante para el desempeño académico. Así, en algunas investigaciones realizadas en carreras de las ciencias administrativas y afines, muestran como las mujeres suelen tener un rendimiento superior al de los hombres (Macan y Dipboye, 1990; González, 1996; Betts y Morrel, 1999; Rodríguez, Fita y Torrado, 2004; Barahona, 2014). Estos estudios argumentan que las mujeres muestran comportamientos más adecuados a las normas universitarias (perseverancia y acatamiento de reglas). Tal vez, las mujeres sean más responsables y por ello su rendimiento sea superior al de los hombres. La literatura muestra a la edad como otra variable no académica que puede determinar el desempeño académico, si bien no existe consenso sobre el sentido de la relación. De este modo, la edad con la que el estudiante accedió a la universidad ha sido identificada por algunos autores como un indicador de rendimiento. Así, esta edad de acceso se puede considerar como un indicador reflejo del rendimiento previo, dado que con este se puede saber si el sujeto ha repetido niveles o ha cursado otra carrera con anterioridad (Montero et al., 2007; Porto y Di Gresia, 2001). Sin embargo, Naylor y Smith (2004) indican lo contrario, ya que concluyen que la edad determina positivamente el desempeño. Los factores culturales y sociales del entorno familiar favorecen el desarrollo intelectual del individuo, por lo general son considerados una forma del denominado capital cultural (Montero, Villalobos y Valverde, 2007). La ciudad de origen del estudiante se podría relacionar eventualmente con el rendimiento académico (Carrión, 2002). En este sentido, Naylor y Smith (2004) han concluido que el residir lejos de donde se encuentra el establecimiento educacional genera un efecto negativo en el desempeño y se condice con los resultados de Di Gresia y Porto en su investigación de 2004, que afirman que el viajar entre las regiones con motivos de estudio genera un impacto negativo en el rendimiento académico. Respecto al colegio de procedencia, este factor se relaciona generalmente con que, si el estudiante proviene de un colegio público o municipal, este obtendría un rendimiento menor al de colegios particulares, esta situación podría asociarse a una mejor preparación académica y mejores oportunidades de aprender de los estudiantes que provienen de colegios particulares (Montero et al., 2007). La investigación realizada en Chile, con el objetivo de detectar los problemas de rendimiento académico, utilizado un Sistema de Medición de Calidad de la Educación (SIMCE), ha mostrado que la formación (de calidad) del estudiante depende en gran medida de su nivel socioeconómico, existiendo una 
diferencia notable en la formación que entregan los colegios privados de élite respecto de los colegios públicos. Estas investigaciones han mostrado que la educación chilena muestra importantes diferencias desde el punto de vista socioeconómico. Por lo que pertenecer a estratos sociales con mayores ingresos garantiza una educación de mayor calidad: Mientras que los estudiantes pertenecientes a familias con ingresos económicos bajos y medios, que acuden a escuelas y colegios que no logran hacer que sus estudiantes alcancen los aprendizajes necesarios para hacer frente a las demandas de la enseñanza universitaria. Esta situación es una característica común en los países Latinoamericanos, donde existen una clase social que no consigue alcanzar los conocimientos necesarios durante la enseñanza secundaria que le permita hacer frente a las demandas de la educación superior (Barahona, 2014). Sin embargo, algunos estudios muestran resultados donde la procedencia del tipo de establecimiento (particular subvencionado o público) al que ha asistido previamente el estudiante no incide en el rendimiento universitario (Barahona, 2014).

Son numerosos los estudios que han identificado las calificaciones académicas procedentes de procesos de evaluación o admisión como un importante predictor del éxito académico de los estudiantes universitarios. Los resultados de las investigaciones muestran como la calificación promedio de la admisión a la universidad es estadísticamente significativa sobre el rendimiento académico de los estudiantes universitarios (Aitken, 1982; Toca y Tourón, 1989; Geiser y Studley, 2001; Díaz et al., 2002; Montero et al. 2007; Rodríguez et al., 2004; Ismail y Othman, 2006; Zeegers, 2007; Etxeberria, 2017). En la misma dirección, algunas investigaciones han concluido que existe una correlación significativa entre las notas medias obtenidas en la educación secundaria y los resultados obtenidos en la universidad, como lo muestran diversos trabajos (Brooks y DuBois, 1995; Betts y Morell, 1999; McKenzie y Schweitzer, 2001; Porto y Di Gresia, 2001; Naylor y Smith, 2004; Vélez y Roa, 2005; Chow, 2010). Otros trabajos muestran evidencias de que el desempeño en el primer año de universidad puede predecir el resultado académico en años posteriores (Larmar y Ingamells, 2010; Trockel, 2010). Porto y Di Gresia (2001) analizaron este efecto en los estudiantes de la cohorte 2000 de la Facultad de Ciencias Económicas de la Universidad Nacional de La Plata, encontrando una influencia positiva y significativa en diversas instancias de la carrera.

Para el caso del ámbito universitario, la literatura revisada muestra conclusiones diversas en función del contexto y/o de las titulaciones estudiadas. Esta diversidad de resultados es comprensible si se considera la variedad de requisitos en cuanto a capacidades que demanda la gran variedad de estudios universitarios existente.

\section{METODOLOGÍA Y RESULTADOS}

En la presente investigación se estudiará la incidencia de diversas variables, académicas y no académicas, en el rendimiento académico y en el tiempo empleado para terminar la carrera por parte de los estudiantes. El objetivo principal de la investigación es determinar y modelar la cantidad de semestres que emplea un estudiante de Contador Auditor de la PUCV (Pontificia Universidad Católica de Valparaíso, Chile) en alcanzar su título universitario, medido a través de diversos factores, relacionados y no con el rendimiento académico.

La muestra utilizada está formada por los 123 estudiantes titulados de la carrera de Contador Auditor de la PUCV, aquellos que han formado parte del actual plan de estudios 
Estudios Pedagógicos XLVII Nº 1: 469-482, 2021

ANÁLISIS DE LOS DETERMINANTES DEL RENDIMIENTO ACADÉMICO. EL CASO DE CONTADOR AUDITOR DE LA PONTIFICIA UNIVERSIDAD CATÓLICA DE VALPARAÍSO

implementado en el año 2009. De este modo, la muestra está formada por todos los egresados desde 2012 hasta 2016.

La duración media que tardan los estudiantes en finalizar sus estudios para este tipo de titulaciones en las universidades chilenas se sitúa en torno a los 12,8 semestres (Informe Anual SIES, 2016). Y concretamente, para la carrera de Contador Auditor en la Pontificia Universidad Católica de Valparaíso, este mismo Informe estima que la duración media de la carrera es de 14,3 semestres, frente a los 10 semestres previstos en el plan de estudios.

Las características de la muestra se recogen en la Tabla 1. La muestra analizada está integrada principalmente por mujeres $(60 \%)$, mayoritariamente $(93 \%)$ por individuos que comenzaron sus estudios universitarios con una edad situada en el intervalo de entre 17 y 19 años. El 33,6\% procedían de Liceo Comercial, y el colegio de procedencia era municipal en el 47,2\% de los casos y particular subvencionado en el $44 \%$ de las ocasiones. Los estudiantes de Contador Auditor de la Escuela de Comercio PUCV tardan en promedio 11,9 semestres en alcanzar su título. Es decir que la diferencia existente entre el plan formal y el plazo real es de 3,9 semestres. Para el caso de los promedios en las áreas de Matemáticas, Finanzas y el Promedio del Primer Año, estas calificaciones son bajo 5,0, siendo en Chile la escala de 1,0 a 7,0. Sobre el lugar de origen de los estudiantes de la carrera de Contador Auditor de la Pontificia Universidad Católica de Valparaíso, éstos provienen en un $78 \%$ de la V Región (lugar donde se encuentra la Institución de Educación Superior objeto de estudio), un 7\% de la Región Metropolitana, un 9\% de la Sexta Región, y el otro 6\% de las otras trece regiones del país.

Tabla 1. Análisis descriptivo de la muestra

\begin{tabular}{|c|c|c|c|}
\hline \multicolumn{2}{|c|}{ Cantidad de semestres } & \multicolumn{2}{|c|}{ Sexo } \\
\hline $\begin{array}{l}\text { Promedio } \\
\text { Desviación Estándar }\end{array}$ & $\begin{array}{c}11,91 \\
1,54\end{array}$ & Mujeres & $56 \%$ \\
\hline Máximo & 16 & Hombres & $44 \%$ \\
\hline \multicolumn{2}{|c|}{ Edad de ingreso } & \multicolumn{2}{|c|}{ Tipo de colegio } \\
\hline 17-19 años & $93 \%$ & Liceo Comercial & $26 \%$ \\
\hline 20 años o más & $7 \%$ & Otro & $74 \%$ \\
\hline \multicolumn{2}{|c|}{ Colegio de procedencia } & \multicolumn{2}{|c|}{ Promedios asignaturas por áreas } \\
\hline Municipal & $32 \%$ & Promedio Matemáticas & 4,7 \\
\hline Particular Subvencionado & $55 \%$ & Promedio Finanzas & 4,3 \\
\hline Particular & $13 \%$ & Promedio Primer Año & 4,7 \\
\hline
\end{tabular}

Fuente. Elaboración propia.

Para modelar la cantidad de semestres que demorará un estudiante de Contador Auditor de la PUCV en alcanzar su título universitario, se especifica un modelo econométrico considerando diversas variables analizadas en la literatura. 
Así, la variable endógena se mide a través de la cantidad de semestres que tarda el estudiante desde su ingreso en la universidad, hasta titularse (cantidad_semestres). De este modo, y de acuerdo a la literatura revisada, la variable cantidad_semestres se utiliza como aproximación a la medición del rendimiento académico (Kuncel, Hezlett y Ones, 2004). Como se ha indicado anteriormente la duración prevista de la titulación en Contador Auditor de la PUCV en el plan de estudios sería de 8 semestres.

Como se ha expuesto previamente, son diversas las variables exógenas o independientes que pueden influir en el rendimiento académico del estudiante. En esta investigación, y siguiendo la literatura, se han agrupado las variables independientes en dos bloques: variables relacionadas con las características no académicas de los estudiantes y variables relativas a las características académicas de los alumnos.

Dentro de las variables relacionadas con las características no académicas de los estudiantes se han considerado la edad de ingreso a la educación superior, siendo la edad mínima de ingreso a la carrera de Contador Auditor en el periodo de análisis de 17 años y la edad máxima de 40 años, y el sexo del estudiante (género). Además, se ha utilizado la región de procedencia del estudiante (región). Esta variable tomará el valor de uno si su región de procedencia coincide con la región donde está ubicada la universidad analizada, esto es, Valparaíso, y cero en caso contrario, es decir si procede de cualquier otra de las 16 regiones del país. Para analizar factores relacionados con aspectos socioeconómicos del estudiante se han utilizado variables que permiten identificar la tipología de colegio de procedencia de los alumnos, en el caso analizado se han identificado, centros municipales, (municipal), centros particulares subvencionados (particular_subvencionado) y establecimientos particulares (particular);

- Por otra parte, en el marco de las variables independientes relacionadas con las características académicas de los estudiantes, se ha considerado las notas que han considerado los siguientes aspectos:

- Las notas de enseñanza media (NEM) obtenidas por los estudiantes, como reflejo y aproximación del rendimiento previo a la universidad. Donde la escala de calificaciones va desde 1,0 a 7,0, siendo el mínimo NEM de 5,0 y el máximo 6,9, para el caso de estudio.

- La puntuación media obtenida por los estudiantes en la Prueba de Selección Universitaria (PSU), lo que contempla los conocimientos previos a la educación superior. Siendo el puntaje mínimo PSU de 533 puntos y el máximo 716 puntos.

- La variable (comercial) que refleja si el estudiante proviene de un colegio técnico comercial o no.

- Variables relacionadas con las notas medias obtenidas por los estudiantes en diversos grupos de materias y en el primer curso académico universitario. Donde la escala de calificaciones para cada uno de los promedios del respectivo grupo de asignaturas va desde 1,0 a 7,0 .

El modelo econométrico propuesto para modelizar el objetivo establecido se estima mediante mínimos cuadrados ordinarios (MCO) de modo que el análisis de los factores que influyen en la cantidad de semestres de los estudiantes de la carrera de Contador Auditor pueda ser expresado en unidades de tiempo (semestres), a través de un modelo lineal. La especificación del modelo queda como se muestra en la ecuación (1). 
Estudios Pedagógicos XLVII Nº 1: 469-482, 2021

ANÁLISIS DE LOS DETERMINANTES DEL RENDIMIENTO ACADÉMICO. EL CASO DE CONTADOR AUDITOR DE LA PONTIFICIA UNIVERSIDAD CATÓLICA DE VALPARAÍSO

cantidad_semestres $_{i}=\beta_{0}+\beta_{1} *$ edad $_{i}+\beta_{2} *$ genero $_{i}+\beta_{3} *$ promedio_finanzas $_{i}+\beta_{4} *$ promedio_matematicas $_{i}$

$+\beta_{5}{ }^{*}$ promedio_economia $_{i}+\beta_{6} *$ promedio_fundamentos $_{i}+\beta_{7}$ ren dimiento_primer $+\beta_{8} * N E M_{i}+\beta_{9} *$ region $_{i}$ (1)

$+\beta_{0} * P S U_{i}+\beta_{1} *$ municipal $_{i}+\beta_{2} *$ particular_subvencionado $_{i}+\beta_{3} *$ particular $_{i}+\beta_{4} *$ comercial $_{i}+\mu_{i}$

En la ecuación (1) se utilizan datos de información de sección cruzada (corte transversal), para poder cuantificar los parámetros del modelo, se utiliza una base de 123 datos de estudiantes que se matricularon entre 2009-2012 y finalizaron sus estudios en el periodo 2012-2016. No se consideran aquellos estudiantes que se han titulado, pero convalidaron uno o más ramos del plan formal, es decir, se consideró la población total de alumnos titulados de forma regular en dicho periodo de tiempo.

Para obtener los datos asociados a cada variable, se utilizó la información proporcionada por la Escuela de Comercio (donde pertenece la carrera de Contador Auditor), de la Pontificia Universidad Católica de Valparaíso, que comprende información actualizada a diciembre de 2016, de todos los alumnos matriculados y titulados de la actual malla curricular implementada desde el año 2009. La estimación del modelo especificado en la ecuación 1 , se presenta en la Tabla 2.

Tabla 2. Estimación de parámetros modelo general

\begin{tabular}{|l|c|c|c|c|}
\hline \multicolumn{1}{|c|}{ Variable } & Coeficientes & $\begin{array}{c}\text { Desviación } \\
\text { Estándar }\end{array}$ & Estadístico $t$ & Probabilidad \\
\hline Edad & $-0,320868$ & 0,124511 & $-2,577030$ & 0,0113 \\
\hline Genero & $-0,434529$ & 0,279937 & $-1,552238$ & 0,1235 \\
\hline Promedio_finanzas & $-1,534963$ & 0,410795 & $-3,736568$ & 0,0003 \\
\hline Promedio_matematicas & $-0,705837$ & 0,233907 & $-3,017600$ & 0,0032 \\
\hline Promedio_economia & 0,019047 & 0,271352 & 0,070192 & 0,9442 \\
\hline Promedio_fundamentos & 0,073030 & 0,295918 & 0,246792 & 0,8055 \\
\hline Rendimiento_primer & $-1,094255$ & 0,469658 & $-1,583878$ & 0,0213 \\
\hline Nem & 0,157030 & 0,488435 & 0,321496 & 0,7485 \\
\hline Region & 0,152576 & 0,305388 & 0,499613 & 0,6184 \\
\hline Psu & 0,012016 & 0,005733 & 2,095857 & 0,0384 \\
\hline Municipal & $-0,370152$ & 0,480512 & $-0,770328$ & 0,4428 \\
\hline Particular_subvencionado & $-0,408225$ & 0,528553 & $-0,772345$ & 0,4416 \\
\hline Particular & 0,211310 & 0,665989 & 0,317288 & 0,7516 \\
\hline Comercial & $-0,160554$ & 0,358493 & $-0,447858$ & 0,6552 \\
\hline C & 19,95612 & 4,173870 & 7,781204 & 0,0000 \\
\hline
\end{tabular}

Fuente. Elaboración propia. 
Posteriormente, y con objeto de seleccionar las variables representativas del avance curricular, se utiliza el método paso a paso, ya que preliminarmente existen variables muy cercanas al nivel de confianza utilizado, las que deberán ser eliminadas del modelo. Respecto a esta selección planteamos un modelo econométrico final que interpreta los factores que determinan el avance curricular de los estudiantes que ingresan a la carrera Contador Auditor. La estimación del modelo especificado anteriormente se presenta en la Tabla 3.

Los resultados presentados en la Tabla 3 nos permiten apreciar niveles de significatividad individual al 90\%, así como también niveles de significancia conjunta del 99\%². La variabilidad de la cantidad de semestres cursados es explicada en un $46 \%$ por la variabilidad de las variables: edad, género, promedio_finanzas, promedio_matematicas, rendimiento_ primer, y PSU. Además, el modelo presenta adecuados criterios de información (Akaike, Schwarz, Hannan-Quinn ${ }^{3}$ ). En cuanto a las hipótesis asociadas a los residuos del modelo, éstas no presentan problemas de autocorrelación de los residuos ${ }^{4}$, tampoco presentan problemas de normalidad de los residuos ${ }^{5}$, como tampoco de heteroscedasticidad de los mismos ${ }^{6}$.

Tabla 3. Estimación de parámetros modelo especificado

\begin{tabular}{|l|c|c|c|c|}
\hline \multicolumn{1}{|c|}{ Variable } & Coeficientes & $\begin{array}{c}\text { Desviación } \\
\text { Estándar }\end{array}$ & Estadístico $\boldsymbol{t}$ & P-valor \\
\hline Edad & $-0,302035$ & 0,119226 & $-2,533288$ & 0,0126 \\
\hline Género & $-0,387044$ & 0,234355 & $-1,651529$ & 0,1013 \\
\hline Promedio_finanzas & $-1,452405$ & 0,322665 & $-4,501281$ & 0,0000 \\
\hline Promedio_matematicas & $-0,686966$ & 0,214266 & $-3,206130$ & 0,0017 \\
\hline Rendimiento_primer & $-1,119327$ & 0,461724 & $-2,429594$ & 0,0173 \\
\hline Psu & 0,013075 & 0,005100 & 2,563679 & 0,0116 \\
\hline C & 19,30463 & 3,624915 & 5,325541 & 0,0000 \\
\hline
\end{tabular}

Fuente. Elaboración propia.

En relación a las variables no académicas se ha identificado como la edad de acceso y el género del estudiante determina la duración de los estudios. Los resultados obtenidos muestran como la mayor edad de ingreso de los estudiantes influye positivamente en la reducción del tiempo que tardan en finalizar sus estudios. Así, por cada año más

\footnotetext{
F-statistic $=12.728 ;$ P-valor $=<0,01$

Akaike: -6,665764; Schwarz: -6.412801; Hannan-Quinn: -6,565058

4 Test de Autocorrelación: Durbin-Watson Test $=2,094, \mathrm{dl}=1,617, \mathrm{du}=1,832, \mathrm{Q}=0,05$

5 Jarque-Bera Test $=1,0067 ;$ P-valor $=0,604488$

$6 \quad$ White Test $=$ F-statistic: 0,$821750 ;$ P-valor $=0,7155$
} 
tenga el estudiante al acceder a la universidad, éste ve disminuir en 0,3 la cantidad de semestres que tardará en finalizar sus estudios en comparación con uno de menor edad. En relación al sexo de los estudiantes, los resultados obtenidos muestran como las mujeres obtienen un mejor rendimiento. En el caso analizado, las mujeres se demoran 0,4 semestres menos que los hombres en terminar la carrera de Contador Auditor, disminuyendo significativamente la brecha en la duración real y formal de la carrera. Como se ha indicado anteriormente, esta situación puede tener su origen en el mayor sentido de la responsabilidad, la perseverancia y el acatamiento de las normas mostrado de las mujeres.

Por el contrario, y si bien la literatura especializada considera el origen socioeconómico y el tipo de establecimiento educacional como variables que pueden influir en el rendimiento académico, los resultados obtenidos en el caso analizado no lo corroboran. El origen socioeconómico del estudiante, aproximado a partir de la tipología de centro donde ha cursado sus estudios previos, no incide en la duración de sus estudios en la carrera de Contador Auditor.

En esta investigación, y siguiendo la literatura, se han agrupado las variables independientes en dos bloques: variables relacionadas con las características no académicas de los estudiantes y variables relativas a las características académicas de los alumnos.

Por otra parte, y en relación a las variables relativas a las características académicas de los alumnos, se ha observado como las puntaciones previas al acceso a la universidad no resultan relevantes, pero si las calificaciones medias obtenidas durante su paso por la misma.

De este modo, las notas de enseñanza media de los estudiantes (NEM), como forma de reflejo del rendimiento previo a la universidad, no resulta tener una incidencia estadísticamente significativa en la duración de la carrera, a pesar de que a priori se pudiera creer que el estudiar en un colegio de tipo comercial y traer cierta base académica especializada mejorase el rendimiento en el estudio de la carrera de Contador Auditor. De este modo, los análisis realizados no han encontrado diferencias estadísticamente significativas que permitan afirmar que estos estudiantes obtendrán un mejor desempeño en la vida universitaria de Contador Auditor.

Otras variables relacionadas con aspectos académicos de los estudiantes han sido identificadas como buenos predictores del desempeño del estudiante. Concretamente las notas medias obtenidas por los estudiantes en las asignaturas de finanzas y matemáticas inciden positiva y significantemente en la reducción del número de semestres necesarios para finalizar los estudios. Por el contrario, las asignaturas de economía y fundamentos de la empresa no son determinantes en la duración real del plan de estudios. Respecto al rendimiento académico de primer año de los estudiantes de la carrera de Contador Auditor de la PUCV, resulta ser un buen predictor de la duración de la carrera, dado que cuando el rendimiento académico de primer año aumenta en un punto, la duración de la carrera disminuye en 1,11 semestres. Por su parte, la PSU nos indica que tiene un impacto positivo en el rendimiento académico, es decir a mejor puntaje de admisión el estudiante se demorará más en titularse. Esta situación, que a prior parece contradictoria, podría deberse a que se utiliza como dato un puntaje ponderado que considera el área de Lenguaje, Matemáticas y Ciencias o Historia, razón por la cual existen riesgos de que esta variable no sea lo suficientemente determinante, como sí lo sería si hubiésemos considerado cada área como una variable por separado. 


\section{CONCLUSIONES}

La presente investigación ha tratado de determinar los factores que son influyentes en el tiempo que invierten los estudiantes en finalizar la titulación de Contador Auditor de la Pontificia Universidad Católica de Valparaíso. Esta información puede resultar de utilidad tanto a los estudiantes a como a la propia institución académica, ya que pueden servirse de estos indicadores para reducir el plazo real de finalización de los estudios al formalmente establecido. Para alcanzar este objetivo, se expresó el rendimiento académico a partir del cálculo de la cantidad de semestres necesarios para finalizar la titulación, entendiendo que esta variable es altamente multicausal y compleja, ya es el resultado de la interacción de distintos factores complejos por sí mismos y que varían de un individuo a otro.

Los análisis realizados han permitido identificar como la variable más influyente al momento de calcular cuánto demorará el estudiante en concluir sus estudios, la nota promedio de las asignaturas de finanzas del plan de estudios, ya a mejor promedio se puede disminuir 1,45 semestres respecto a un alumno con menor rendimiento. Sin perjuicio de lo anterior, la nota promedio de las asignaturas de matemáticas del plan curricular, es el que tiene una mayor correlación respecto al tiempo que tomará un estudiante en titularse, permitiéndole disminuir 0,69 semestres respecto a un estudiante con un promedio de notas menor.

Así mismo la nota de acceso a la universidad aumenta la cantidad de semestres marginalmente, y el género, que también influye, es el que menos aporta a la variabilidad de la duración de la carrera. Sin perjuicio de lo anterior, la cantidad de semestres disminuye si el estudiante es mujer. Si el estudiante tiene mayor edad al ingresar, este podrá disminuir en 0,3 semestres la duración de su carrera, respecto a aquellos que ingresan a menor edad.

Otro punto importante es que, el paradigma del origen socioeconómico y el desempeño en la educación media, que serían factores relevantes al pronosticar el resultado de un estudiante que ingresa a la universidad, para el caso de la carrera de Contador Auditor de la Pontificia Universidad Católica de Valparaíso, no corresponderían a factores predictivos significantes en el contexto de esta investigación, si bien dicha variables puede ser predictora válida en otras carreras universitarias, en otras universidades, en general en otros contextos.

Según todos los resultados descritos, se recomienda que el estudiante pueda mejorar o poner sus esfuerzos en aquellas variables más importantes y significativas para el rendimiento académico, tales como las áreas disciplinares de finanzas y matemáticas, las que proporcionan una mejor predicción en el tiempo de duración de sus estudios, pero sin descuidar las otras áreas académicas que resultaron no ser significantes para esta investigación.

En cuanto a la Escuela de Comercio, unidad donde pertenece la carrera de Contador Auditor de la PUCV, se espera que las conclusiones a las que ha llegado la presente investigación, puedan servir como base para futuros análisis y/o modificaciones del plan de estudios considerando fundamental el alcanzar ser un soporte al acompañamiento de los estudiantes en esta etapa de adaptación como lo es el primer año; presentarle a los estudiantes las oportunidades que se presentan cuando la persona tiene constancia en el estudio, y, fomentar la lealtad hacia la institución, creando una buena imagen y retención a partir de un trabajo académico responsable.

Y en un contexto más general, la identificación de los factores asociados al rendimiento académico puede permitir a las autoridades universitarias diseñar políticas que permitan mejorar la calidad académica de los estudiantes de pregrado. 
Estudios Pedagógicos XLVII Nº 1: 469-482, 2021

ANÁLISIS DE LOS DETERMINANTES DEL RENDIMIENTO ACADÉMICO. EL CASO DE CONTADOR AUDITOR DE LA PONTIFICIA UNIVERSIDAD CATÓLICA DE VALPARAÍSO

Este trabajo, puede servir de punto de partida de una investigación más amplia. Por un lado, ampliar el número de alumnos analizado incorporado otras cohortes de egresados, lo que además permitiría poder hacer vario estudios transversales e identificar los posibles cambios en las variables predictoras que pudieran producirse. Además, esta investigación se podría desarrollar en otras titulaciones y universidades, lo que además permitiría estudios comparados.

\section{REFERENCIAS BIBLIOGRÁFICAS}

Aitken, N. (1982). College student performance, satisfaction, and retention: Specification and estimation of a structural model. Journal of Higher Education, 53(1), 32-50. https://doi.org/10. 1080/00221546.1982.11780423

Améstica, L., Llinas, X. \& Escardíbul, J., (2017). Costos de la renovación curricular. Una propuesta metodológica para la valorización económica de carreras universitarias. Formación Universitaria, 10(1), 89-100. http://dx.doi.org/10.4067/S0718-50062017000100010

Barahona U. P. (2014). Factores determinantes del rendimiento académico de los estudiantes de la Universidad de Atacama. Estudios Pedagógicos, 40(1), 25-39. http://dx.doi.org/10.4067/S071807052014000100002.

Betts, J. R. \& Morell, D. (1999). The determinants of undergraduate grade point average: The relative importance of family background, high school resources, and peer group effects. The Journal of Human Resources, 34(2), 268-293. https://doi.org/10.2307/146346

Brooks, J. H. \& DuBois, D. L. (1995). Individual and environmental predictors of adjustment during the first year of college. Journal of College Student Development, 36(4), 347-360.

Carrión Pérez, E. (2002). Validación de características al ingreso como predictores del rendimiento académico en la carrera de medicina. Educación Médica Superior, 16(1), 1-2.

Chow, H. P. H. (2010). Predicting academic success and psychological wellness in a sample of Canadian undergraduate students. Electronic Journal of Research in Educational Psychology, 8(2), 473-496.

Clark, E. \& Ramsay, W. (1990). Problems of retention in tertiary education. Education Research and Perspectives, 17(2), 47-59.

Combey, P. (1993). The British system of engineering higher education: an example of a system undergoing innovation under pressure. European Journal of Engineering Education, 18(1), 5164. https://doi.org/10.1080/03043799308923209

Córdoba Caro, L. G., García Preciado, V., Luengo Pérez, L. M., Vizuete Carrizosa, M. \& Molina, S. (2011). Determinantes socioculturales: su relación con el rendimiento académico en alumnos de Enseñanza Secundaria Obligatoria. Revista de Investigación Educativa, 29(1), 83-96.

Di Gresia, L. M. \& Porto, A. (2004). Dinámica del desempeño académico. Documentos de Trabajo. ISSN: $1853-3930$.

Díaz, M., Peio, A., Arias, J., Escudero, T., Rodríguez, S. \& Vidal, G. J. (2002). Evaluación del Rendimiento Académico en la Enseñanza Superior. Comparación de resultados entre alumnos procedentes de la LOGSE y del COU. Revista de Investigación Educativa, 2(20), 357-383.

Edel Navarro, R. (2003). El Rendimiento Académico: Concepto, Investigación y Desarrollo. Revista Electrónica Iberoamericana sobre Calidad, Eficacia y Cambio en Educación, 1(2). Última visita 11 de junio de 2019. Recuperado desde: https://revistas.uam.es/index.php/reice/article/ view/5354/5793.

Espinoza, R. \& Urzúa, S. (2015). Las Consecuencias Económicas de un Sistema de Educación Superior Gratuito en Chile. Revista de educación, 370, octubre-diciembre. 10-44. doi: 10.4438/1988-592X-RE-2015-370-295 
Estudios Pedagógicos XLVII N 1: 469-482, 2021 ANÁLISIS DE LOS DETERMINANTES DEL RENDIMIENTO ACADÉMICO. EL CASO DE CONTADOR AUDITOR DE LA PONTIFICIA UNIVERSIDAD CATÓLICA DE VALPARAÍSO

Etxeberria, P., Alberdi, E., Eguia, I. \& García, M. J., (2017). Análisis del Rendimiento Académico en Relación al Perfil de Ingreso del Alumnado e Identificación de Carencias Formativas en Materias Básicas de dos Grados de Ingeniería. Formación Universitaria, 10(4), 67-74. doi: 10.4067/ S0718-50062017000400007.

Fernández González, O., Martínez-Conde Beluzan, M. \& Melipillán Araneda, R. (2018). Estrategias de aprendizaje y autoestima. Su relación con la permanencia y deserción universitaria. Estudios Pedagógicos, 35(1), 27- 45. doi: 10.4067/S0718-07052009000100002

Garbanzo, G. M. (2007). Factores asociados al rendimiento académico en estudiantes universitarios, una reflexión desde la calidad de la educación superior pública. Revista Educación, 31(1), 4363. DOI: 10.15517/REVEDU.V31I1.1252

Geiser, S. \& Studley, R. (2001). UC and the SAT: Predictive Validity and Differential Impact of the SAT I and SAT II at the University of California. University of California, Office of the President

González Fiegehen, L. E. (2007). Repitencia y deserción universitaria en América Latina. En Informe sobre la educación superior en América Latina y el Caribe2000-2005. Capítulo 11, 156-168. Última visita 11 de junio de 2019. Recuperado desde: https://www.oei.es/historico/salactsi/ informe_educacion_superiorAL2007.pdf

González López, I. (2004). Realización de un análisis discriminante explicativo del rendimiento académico en la universidad. Revista de Investigación Educativa, 22(1), 43-59.

González, F. (1996). Comprensión lectora y rendimiento académico. Revista Gallega de Psicopedagogía, 13(9), 209-221.

Herrera, C.; Pérez, C. \& Echeita, G. (2016). Teorías implícitas y prácticas de enseñanza que promueven lainclusión educativa en la universidad. Instrumentos y antecedentes para la reflexión y discusión. Formación Universitaria, 9(5), 49-64. doi: 10.4067/S0718-50062016000500006.

Himmel, E. (2002). Modelos de análisis de la deserción estudiantil en la Educación Superior. Revista Calidad de la Educación Superior, (17), 91-108. doi: http://dx.doi.org/10.31619/caledu.n17.409 . (2003). Evaluación de aprendizajes en la Educación Superior: Una reflexión necesaria. Pensamiento Educativo, 33, 199-211.

Ismail, N. A. \& Othman, A. (2006). Comparing university academic performances of HSC students at the three art-based faculties. International Education Journal, 7(5), 668-675.

Kuncel, N. R., Hezlett, S. A. \& Ones, D. S. (2004). Academic performance, career potential, creativity, and job performance: can one construct predict them all? Journal of Personality and Social Psychology, 86(1), 148-161. https://doi.org/10.1037/0022-3514.86.1.148

Larmar, S. \& Ingamells, A. (2010). Enhancing the first-year university experience: linking university orientation and engagement strategies to student connectivity and capability. Research in Comparative and International Education, 5(2), 210-223. doi: 10.2304/rcie.2010.5.2.210

Macan, T. \& Dipboye, R. (1990). College students' time management: correlations with academic performance and stress. Journal of Educational Psychology, 82(4) 760-768. https://doi. org/10.1037/0022-0663.82.4.760

Mateo-Canedo, C., Canet-Juric, L. \& Andrés, M. L. (2015). Deserción y lentificación en los estudios universitarios: aportes cognitivos para un mejor rendimiento académico. Question, 48(1), 147159.

McKenzie, K. \& Schweitzer, R. (2001). Who succeeds at University? Factors predicting academic performance in first year Australian university students. Higher Education Research \& Development, 20(1), 21-33. https://doi.org/10.1080/07924360120043621

Montero, E., Villalobos, J. \& Valverde, A. (2007). Factores institucionales, pedagógicos, psicosociales y sociodemográficos asociados al rendimiento académico en la Universidad de Costa Rica: Un análisis multinivel. Revista Electrónica de Investigación y Evaluación Educativa, 13(2), 215-234.

Naylor, R. \& Smith, J. (2004). Determinants of educational success in Higher education, en G. Johnes y J. Johnes (Ed.), International Handbook in the economics of education (pp. 415-461). UK: E. E. Publishing. doi: 10.4337/9781845421694.00016 
Palominos, F., Díaz, H., Palominos, S. \& Canete L. (2018). Relación entre los procedimientos de selección a la educación superior y el desempeño académico de los estudiantes con base en una clasificación mediante conjuntos difusos. Formación Universitaria, 11(1), 45-52. doi: 10.4067/ S0718-50062018000100045

Pérez-Luño, A., Jerónimo, R. J. \& Sánchez-Vázquez, J. (2000). Análisis exploratorio de las variables que condicionan el rendimiento académico. Sevilla, España: Universidad Pablo de Olavide.

Porto, A. \& Di Gresia, L. (2001). Rendimiento de estudiantes universitarios y sus determinantes. Revista de Economía y Estadística, 42(1), 93-113.

Quispe, J. T. (2010). Factores que influyen en el rendimiento académico y la deserción de los estudiantes de la facultad de ingeniería económica de la Una-Puno, periodo 2009, Cuadernos de Educación y Desarrollo, 2(11).

Rodríguez, S., Fita, E. \& Torrado, M. (2004). El rendimiento académico en la transición secundariauniversidad. Revista de Educación, 334, 391-414.

Sayans-Jiménez, P., Vázquez-Cano, E. \& Bernal-Bravo, C. (2018). Influencia de la riqueza familiar en el rendimiento lector del alumnado en PISA. Revista de Educación, 380 (abril-junio). 129155. doi: 10.4438/1988-592X-RE-2017-380-375.

Sebastián, C. (2007). La diversidad interindividual como una oportunidad para el aprendizaje de los estudiantes de educación superior. Calidad en la Educación, 26, 83-101. doi: 10.31619/caledu. $\mathrm{n} 26.234$

Servicio de Información de Educación Superior (SIES) (2016). Informe anual "Duración real y Sobreduración de las carreras de Educación Superior 2011-2015”. Última visita 11 de junio de 2019. Recuperado desde http://www.mifuturo.cl/images/Informes_sies/Duracion_carreras/ duracion\%20real\%20y\%20sobredurasob_sies_2016.pdf

Toca, M. T. \& Tourón, J. (1989). Factores del rendimiento académico en los estudios de arquitectura. Revista de Investigación Educativa, 14(7), 31-45.

Tomás-Miquel, J. V., Expósito-Langa, M. \& Sempere-Castelló, S. (2014). Determinantes del rendimiento académico en los estudiantes de grado. Un estudio en administración y dirección de empresas. Revista de Investigación Educativa, 32(2), 379-392. doi: 10.6018/rie.32.2.177581

Trockel, M., Barnes, M. \& Egget, D. (2010). Health-related variables and academic performance among first-year college students: implications for sleep and other behaviors. Journal of American College Health, 49(3), 125-131. doi: 10.1080/07448480009596294

Vélez Van, A. \& Roa, C. (2005). Factors associated with academic performance in medical students. Educación Médica, 8(2), 74-82.

Yáñez, L. \& Soria, K. (2017). Reflexión de buenas prácticas docentes como eje de calidad en la educación universitaria: caso escuela de ciencias empresariales de la Universidad Católica del Norte. Formación Universitaria, 10(5), 59-68. doi: /10.4067/S0718-50062017000500007.

Zeegers, P. (2007). Student learning in higher education: a path analysis of academic achievement in science. Higher Education Research \& Development, 23(1), 35-56. https://doi. org/10.1080/0729436032000168487 